\title{
Dividing by Demanding: \\ Object Division through Market Procedures
}

\author{
Claus-Jochen Haake \\ Institute of Mathematical Economics, Bielefeld University \\ P.O. Box 100131, 33501 Bielefeld, Germany \\ and \\ Department of Mathematics, Harvey Mudd College, \\ Claremont, USA \\ email: chaake@wiwi.uni-bielefeld.de
}

July 2004

\begin{abstract}
We discuss a model, in which two agents may distribute finitely many objects among themselves. The conflict is resolved by means of a market procedure. Depending on the specifications, this procedure serves to implement bargaining solutions such as the discrete Raiffa solution, the Kalai-Smorodinsky solution and the Perles-Maschler solution. The latter is axiomatized using the superadditivity axiom, which in the present context is readily interpreted as resolving a specific source of conflict potential.

Keywords: Object Division, Market Procedure, Perles-Maschler Solution

JEL Classification: C78, C62, D51, D63

I would like to thank Joachim Rosenmüller, Francis Su and Walter Trockel for fruitful discussions and the members of the Harvey Mudd College in Claremont for making it a great place to work. Financial support from the project "Fairness und Anreize", FiF-Projekt, Bielefeld University is gratefully acknowledged.
\end{abstract}




\section{Introduction}

Probably the most frequent source for two person bargaining problems are object division problems. Two agents are entitled to divide a set of objects among themselves. Examples are easily found in divorce cases or the division of real estate among two heirs. Although seemingly different, the scenario in which two parties bargain over different issues, each of which can be solved by choosing one option, also falls in the class of problems we want to study.

The literature offers a couple of solution procedures to solve this kind of conflict. Thereby, different procedures implement solutions that exhibit different properties. For example, the "Adjusted Winner" Procedure as discussed in Brams \& Taylor (1996) yields an equitable and envy-free solution. It implements the axiomatic bargaining solution introduced by Kalai \& Smorodinsky (1975).

While most existing procedures start with an allocation of objects that is modified during the course of the procedure, the aim in this paper is to determine allocations by means of a market procedure. Roughly spoken, this means that we want to resolve the object division conflict by letting both agents choose their bundle of objects separately. For this, we attach prices to the different objects, endow agents with initial income and then let them spend their incomes on the different items. the advantage is that once prices are fixed, the agents do not have to enter another round of negotiation. In fact, there is a decentralization of the conflict through prices. The final allocation is simply achieved after a utility maximization exercise.

Clearly, the first question to be asked is how to set the prices that this procedure works. Here it turns out that object prices have to satisfy a simple condition that ensures that there will be no further conflict about who will receive which item. In other words, such prices constitute Walrasian equilibrium prices in a derived market. And, since the allocation that prevails will be a Walrasian equilibrium, it constitutes an efficient allocation of the objects.

A second goal in the paper is to use this market procedure to implement known axiomatic bargaining solutions. This can be accomplished by choosing the right prices and applying the procedure. As we will see, this may involve iteration. But still, once an object is allocated to some party, it will stay there during the course of action. We are able to provide implementation results for the Kalai-Smorodinsky solution, the discrete Raiffa solution and the solution by Perles \& Maschler (1981b). The latter is axiomatized by the superadditivity axiom, which has an appealing interpretation in our context. It says that neither of the parties is willing to split the whole set of objects into smaller ones and proceed over each of them separately, since there will be no benefits for neither one. So, the superadditivity notion removes one potential source of conflict, i.e. the question how to proceed. Surprisingly, this solution is implementable by applying the market procedure only once. 
The paper is organized as follows: Section 2 provides the basic framework and discusses demand correspondences. In Section 3 we discuss the condition that ensures existence of Walrasian equilibria. The basic market procedure as well as three of its specifications are presented in Section 4. Here implementation results appear. Section 5 concludes.

\section{The Framework}

We consider a fair division problem in which two agents may divide a finite list of (desirable) objects (goods) among themselves. Denote by $\mathcal{K}:=\{1, \ldots K\}$ the set of objects. Although we think of the objects as indivisible ones, we will assume that they are perfectly divisible. However, it is well known, that in our setup any efficient division of $\mathcal{K}$ involves a split of at most one object.

Therefore, we will also consider the set $\underline{X}^{\mathcal{K}}:=[0,1]^{K}$ of assignments to an agent. Furthermore, we assume that preferences over (complete) objects can be represented by vNM utility functions $u^{i}: \mathcal{K} \longrightarrow \mathbb{R}(i=1,2)$ and that both agents have linear utilities over divisions of an object. Finally, we want to assume that utility functions are additively separable across goods. These assumptions determine agents utility functions on $\underline{X}^{\mathcal{K}}$, which should be expressed by mappings $U^{i}: \underline{X}^{\mathcal{K}} \longrightarrow \mathbb{R} \quad(i=1,2)$ with $U^{i}\left(x^{i}\right):=\sum_{k \in \mathcal{K}} u^{i}(k) x_{k}^{i} \quad\left(x^{i} \in \bar{X}^{\mathcal{K}}\right)$, which reflect $i$ 's utility over assignments.

An allocation is a pair $\left(x^{1}, x^{2}\right) \in \underline{X}^{\mathcal{K}} \times \underline{\bar{X}}^{\mathcal{K}}$ of assignments such that $\sum_{i=1,2} x_{k}^{i}=1$ for each $k \in \mathcal{K}$ holds true. Let $\Delta$ denote the set of price systems $p \in \mathbb{R}_{+}^{K}$ satisfying $\sum_{k \in \mathcal{K}} p_{k}=1$. Then the value of an assignment $x^{i}$ under price system $p$ is the inner product $p \cdot x$.

We start with agent $i$ 's demand correspondence which is defined by $\varphi: \Delta \times \mathbb{R}_{+} \Longrightarrow \underline{X}^{\mathcal{K}}$ with

$$
\varphi^{i}(p, m)=\arg \max \left\{\sum_{k \in \mathcal{K}} u^{i}(k) x_{k}^{i} \mid x^{i} \in \underline{\bar{X}}^{\mathcal{K}}, p \cdot x^{i} \leq m\right\} \quad(i=1,2),
$$

where $m$ corresponds to the agent's endowment in "money / value". In the present context, agent $i$ 's maximization problem is solved by first ordering the objects in $\mathcal{K}$ by their ratios $u^{i}(k) / p_{k}$, which reflect "utility per dollar". Then agent 1 demands the objects according to such an order until his budget is exhausted. Here linearity guarantees that an agent prefers to obtain an object entirely, before he wants to have (fractions of another one).

Denote by $S_{\mathcal{K}}$ the set of all permutations of goods, i.e. all bijective mappings from $\mathcal{K}$ onto $\mathcal{K}$. 
Let

$$
\Pi^{i}(p):=\left\{\pi \in S_{\mathcal{K}} \mid \frac{u^{i}\left(\pi^{-1}(j)\right)}{p_{\pi^{-1}(j)}} \geq \frac{u^{i}\left(\pi^{-1}(j+1)\right)}{p_{\pi^{-1}(j+1)}}(j=1, \ldots, K-1)\right\}
$$

be the set of permutations, such that agent $i$ 's utilities per dollar at prices $p$ are ordered nonincreasingly. Note that $\Pi^{i}(p)$ contains more than one element, if there are at least two goods with equal ratios. Yet, any two elements $\pi, \pi^{\prime} \in \Pi^{i}(p)$ are equivalent in the sense that they satisfy the condition

$$
\frac{u^{i}\left(\pi^{-1}(j)\right)}{p_{\pi^{-1}(j)}}>\frac{u^{i}\left(\pi^{-1}(k)\right)}{p_{\pi^{-1}(k)}} \Longleftrightarrow \quad \frac{u^{i}\left(\pi^{\prime-1}(j)\right)}{p_{\pi^{\prime-1}(j)}}>\frac{u^{i}\left(\pi^{\prime-1}(k)\right)}{p_{\pi^{\prime-1}(k)}}
$$

for all $j, k \in \mathcal{K}$.

To compute agent $i$ 's demand along the order $\pi \in \Pi^{i}(p)$ with budget $m$, one can determine the maximal number $J^{\pi}$ such that $\sum_{j=1}^{J^{\pi}} p_{\pi^{-1}(j)} \cdot 1 \leq m$ is satisfied. That is, agent $i$ can afford objects $\pi^{-1}(1)$ to $\pi^{-1}\left(J^{\pi}\right)$ and will spend the remaining money to buy a fraction of object $\pi^{-1}\left(J^{\pi}+1\right)$. So, his demand for each object $k \in \mathcal{K}$ at order $\pi$ is given by

$$
\left(\varphi^{i, \pi}(p, m)\right)_{k}=1_{\left\{\pi^{-1}(1), \ldots, \pi^{-1}\left(J^{\pi}\right)\right\}}(k)+\frac{m-\sum_{j=1}^{J^{\pi}} p_{\pi^{-1}(j)}}{p_{\pi^{-1}\left(J^{\pi}+1\right)}} \cdot 1_{\left\{\pi^{-1}\left(J^{\pi}+1\right)\right\}}(k) \quad(k \in \mathcal{K}) .
$$

Since $\Pi^{i}(p)$ is not necessarily single valued, we have as demand correspondence

$$
\varphi^{i}(p, m)=\left\{\varphi^{i, \pi}(p, m) \mid \pi \in \Pi^{i}(p)\right\} .
$$

Any object division problem generates a two-person bargaining problem in the following way: Status quo points will always be the origin. For $k \in \mathcal{K}$ let $V^{k}:=\operatorname{comp} H\left(\operatorname{cvH}\left(\left\{\left(u^{1}(k), 0\right),\left(0, u^{2}(k)\right)\right\}\right)\right)$ be the set of possible utility allocations arising from the division of good $k$. This includes the possibility of free disposal. With additive separability of utilities, the bargaining problem arising from an object division problem is represented by the $\operatorname{sum} V=\sum_{k \in \mathcal{K}} V^{k}$. We will denote the class of associated bargaining problems by $\mathcal{U}$. Note that the class of bargaining problems generated by object division problems is the class of compactly generated polyhedral games. See, for example, Perles \& Maschler (1981b) or Rosenmüller (2000). Conversely, any polyhedral bargaining game canonically corresponds to a market, in which no two goods $j$ and $k$ admit of the same ratio, i.e., $\frac{u^{i}(j)}{u^{i}(k)}=\frac{u^{3-i}(j)}{u^{3-i}(k)}$. This market shall be called standard representation (of $V \in \mathcal{U})$.

We will call a mapping $\varphi: \mathcal{U} \longrightarrow \mathbb{R}^{2}$ a bargaining solution on $\mathcal{U}$, if for every $V \in \mathcal{U}$ the point $\varphi(V)$ is Pareto efficient and individual rational (i.e. here, $\varphi(V) \geq 0$ ). Moreover $\varphi$ is supposed to satisfy the symmetry axiom and be covariant with affine transformations of utility.1

\footnotetext{
${ }^{1}$ For a discussion of these axioms see,e.g., Peters (1992).
} 


\section{$3 \quad$ Walrasian Equilibria}

To analyze the object division problem, we set up a two-agent Walrasian exchange economy $\mathcal{E}$ for the objects in $\mathcal{K}$. Thus, the commodity space is $\underline{\bar{X}}^{\mathcal{K}}$. Agents' preferences are represented by the utility functions $U^{i}(i=1,2)$. Initial endowments are denoted by $\left(\omega^{1}, \omega^{2}\right) \in[0,1]^{K} \times[0,1]^{K}$. Thus, the tuple $\mathcal{E}=\left(\mathcal{K}, U^{1}, U^{2}, \omega^{1}, \omega^{2}\right)$ describes the economy.

Note that existence of Walrasian equilibria in $\mathcal{E}$ is guaranteed, since utility functions are in particular concave. Moreover, due to monotonicity of utility functions, any Walrasian equilibrium is efficient according to the First Welfare Theorem.

Define an operation ${ }^{*}: S_{\mathcal{K}} \longrightarrow S_{\mathcal{K}}$ by $\pi \mapsto \pi^{*}$ with $\pi^{*}(l):=K+1-\pi(l)$. This operation simply "reverses the order" in $\pi$. By straightforward computations, one checks $\pi^{*-1}(q)=$ $\pi^{-1}(K+1-q)$.

The market clearing condition in equilibrium can be established for all price systems, such that the orders according to which agents demand goods are inverse to each other. Such a price will then establish an equilibrium price, which is independent of the initial endowment.

To make this precise, we introduce the following condition:

\section{Definition 1}

(i) For $i=1,2, j, k \in \mathcal{K}$ with $u^{i}(k) \neq 0$ denote by $s_{j k}^{i}:=\frac{u^{i}(j)}{u^{i}(k)}$ agent $i$ 's substitution rate between objects $j$ and $k$.

(ii) We say $p \in \Delta$ satisfies the reversed order condition (REVORD), if

$$
\frac{p_{j}}{p_{k}} \in\left[\min \left(s_{j k}^{1}, s_{j k}^{2}\right), \max \left(s_{j k}^{1}, s_{j k}^{2}\right)\right]
$$

holds for all $j, k \in \mathcal{K}$ with $p_{k}, u^{1}(k), u^{2}(k) \neq 0$.

Then we can show that REVORD induces reversed orders.

\section{Lemma 1}

A price system $\bar{p} \in \Delta$ satisfies REVORD if and only if there exists a pair $\left(\pi^{1}, \pi^{2}\right) \in \Pi^{1}(\bar{p}) \times \Pi^{2}(\bar{p})$ with $\pi^{1}=\left(\pi^{2}\right)^{*}$.

\section{Proof:}

Step 1 Necessity: If $\bar{p}=\lambda u^{i}(k) \in \Delta$ for all $k \in \mathcal{K}$, appropriate $\lambda>0$ and some $i$ then 
the assertion is obvious, since here $\Pi^{i}(\bar{p})=S_{\mathcal{K}}$ holds. Suppose that this is not the case and Assume there is no such pair as claimed. Then for every $\pi \in \Pi^{1}(\bar{p})$ there exist $j^{\prime}, k^{\prime} \in \mathcal{K}$ with $\pi^{*}\left(j^{\prime}\right)>\pi^{*}\left(k^{\prime}\right)$ and

$$
\frac{u^{2}\left(j^{\prime}\right)}{u^{2}\left(k^{\prime}\right)}=s_{j^{\prime} k^{\prime}}^{2}>\frac{\bar{p}_{j^{\prime}}}{\bar{p}_{k^{\prime}}}, \quad \frac{u^{1}\left(j^{\prime}\right)}{u^{1}\left(k^{\prime}\right)}=s_{j^{\prime} k^{\prime}}^{1}>\frac{\bar{p}_{j^{\prime}}}{\bar{p}_{k^{\prime}}}
$$

holds. The assumption $\bar{p} \neq \lambda u^{i}(\cdot)$ guarantees that strict inequality holds for some $\pi \in \Pi^{1}(\bar{p})$. The two inequalities imply $\frac{\bar{p}_{j^{\prime}}}{\bar{p}_{k^{\prime}}}<\min \left(s_{j^{\prime} k^{\prime}}^{1}, s_{j^{\prime} k^{\prime}}^{2}\right)$ which contradicts REVORD. Step 2: Suppose REVORD is not satisfied. Then there exist $\hat{j}, \hat{k} \in \mathcal{K}$ with

$$
\text { (i) } \frac{\bar{p}_{\hat{j}}}{\bar{p}_{\hat{k}}}<s_{\hat{j} \hat{k}}^{1}=\frac{u^{1}(\hat{j})}{u^{1}(\hat{k})}, \quad \text { (ii) } \frac{\bar{p}_{\hat{j}}}{\bar{p}_{\hat{k}}}<s_{\hat{j} \hat{k}}^{2}=\frac{u^{2}(\hat{j})}{u^{2}(\hat{k})}
$$

(or analogously with both strict inequalities in the reverse direction). For $\pi \in \Pi^{1}(\bar{p})$, (i) implies $\pi(\hat{j})<\pi(\hat{k})$. Hence, $\pi^{*}(\hat{j})=K+1-\pi(\hat{j})>K+1-\pi(\hat{k})=\pi^{*}(\hat{k})$ follows, which in view of (ii) shows $\pi^{*} \notin \Pi^{2}(\bar{p})$. This shows that there is no pair $\left(\pi, \pi^{*}\right) \in \Pi^{1}(\bar{p}) \times \Pi^{2}(\bar{p})$.

Lemma 1 states that the orders according to which agents demand their objects are inverse, if and only if price ratios are always located between the agents' substitution rates. By "inverse" we mean that we could line up the objects and agent 1 starts to demand on the left whereas agent 2's demand goes from right to left. Thus, there is (up to indifferences) no contradiction on how to distribute the objects in an exchange economy.

\section{Theorem 1}

Let $\mathcal{E}$ be an exchange economy as above and let $\bar{p} \in \Delta$ satisfy REVORD.

Then for any initial endowment $\left(\omega^{1}, \omega^{2}\right)$ there exists an allocation $\bar{x}$ in $\mathcal{E}$ such that $\bar{x}^{i} \in$ $\varphi^{i}\left(\bar{p}, \bar{p} \omega^{i}\right)$. Hence, the pair $(\bar{x}, \bar{p})$ constitutes a Walrasian equilibrium. In particular, $\bar{p}$ is an equilibrium price vector, independent of initial endowments.

\section{Proof:}

Let $\bar{p}$ satisfy REVORD. Take an arbitrary $\pi \in \Pi^{1}(\bar{p})$ with $\pi^{*} \in \Pi^{2}(\bar{p})$. We show that markets are cleared under prices $\bar{p}$ and along orders $\left(\pi, \pi^{*}\right)$ (cf. (1)). According to $\left(\pi, \pi^{*}\right)$ the aggregate 
demand (for all goods) is $2^{2}$

$$
\begin{aligned}
& \varphi^{1, \pi}\left(\bar{p}, \bar{p} \omega^{1}\right)+\varphi^{2, \pi^{*}}\left(\bar{p}, \bar{p} \omega^{2}\right) \\
& =\sum_{j=1}^{J^{\pi}} e^{\pi^{-1}(j)}+\frac{\bar{p} \omega^{1}-\sum_{j=1}^{J^{\pi}} \bar{p}_{\pi^{-1}(j)}}{\bar{p}_{\pi^{-1}\left(J^{\pi}+1\right)}} e^{\pi^{-1}\left(J^{\pi}+1\right)}+\sum_{l=1}^{N^{\pi}} e^{\pi^{*-1}(l)}+\frac{\bar{p} \omega^{2}-\sum_{l=1}^{N^{\pi}} \bar{p}_{\pi^{*-1}(l)}}{\bar{p}_{\pi^{*-1}\left(N^{\pi}+1\right)}} e^{\pi^{*-1}\left(N^{\pi}+1\right)} \\
& =\sum_{j=1}^{J^{\pi}} e^{\pi^{-1}(j)}+\sum_{l=K+1-N^{\pi}}^{K} e^{\pi^{-1}(l)}+\frac{\bar{p} \omega^{1}-\sum_{j=1}^{J^{\pi}} \bar{p}_{\pi^{-1}(j)}}{\bar{p}_{\pi^{-1}\left(J^{\pi}+1\right)}} e^{\pi^{-1}\left(J^{\pi}+1\right)}+\frac{\sum_{l=K+1-N^{\pi}}^{K} \bar{p}_{\pi^{-1}(l)}}{\bar{p}_{\pi^{-1}\left(K-N^{\pi}\right)}} e^{\pi^{-1}\left(K-N^{\pi}\right)} .
\end{aligned}
$$

So, agent 1 demands goods $\pi^{-1}(1), \ldots, \pi^{-1}\left(J^{\pi}\right)$ completely and spends his remaining income on object $\pi^{-1}\left(J^{\pi}+1\right)$. Analogously, $N^{\pi}$ is given for the second agent. We have to show that either $J^{\pi}+N^{\pi}=|K|\left(\right.$ in case $\left.\bar{p} \omega^{1}-\sum_{j=1}^{J^{\pi}} \bar{p}_{\pi^{-1}(j)}=\bar{p} \omega^{2}-\sum_{l=1}^{N^{\pi}} \bar{p}_{\pi^{-1}(l)}=0\right)$ or $J^{\pi}+N^{\pi}=|K|-1$ (in the remaining case) holds true ${ }^{3}$.

Suppose to the contrary $J^{\pi}+N^{\pi}>\mathcal{K}$. Then $\varphi^{1, \pi}\left(\bar{p}, \bar{p} \omega^{1}\right)+\varphi^{2, \pi^{*}}\left(\bar{p}, \bar{p} \omega^{2}\right)>(1, \ldots, 1)$ (strictly at least in coordinate $\left.J^{\pi}\right)$. Therefore, $\bar{p}\left(\varphi^{1, \pi}\left(\bar{p}, \bar{p} \omega^{1}\right)+\varphi^{2, \pi^{*}}\left(\bar{p}, \bar{p} \omega^{2}\right)\right)>\bar{p}\left(\omega^{1}+\omega^{2}\right)$ which contradicts at least one budget constraint.

The remaining cases can be excluded using similar arguments. Observe that for any $\pi \in \Pi^{1}(\bar{p})$ (with $\left.\pi^{*} \in \Pi^{2}(\bar{p})\right)$ the allocation $\bar{x}=\left(\varphi^{1, \pi}\left(\bar{p}, \bar{p} \omega^{1}\right), \varphi^{2, \pi^{*}}\left(\bar{p}, \bar{p} \omega^{2}\right)\right)$ is an equilibrium allocation.

Clearly, a converse of Theorem 1 in the sense that for given $\omega$ only REVORD prices constitute equilibrium prices is not true. According to Lemma 1 condition REVORD is equivalent to obtaining orders of demand that are inverse to each other. Yet, excess demand (which depends on $p$ ) is zero as long as there are no inconsistencies in the orders up to the place, where budgets are spent. For those objects that are not demanded due to budget constraints, a consistency of the ordering is not required. Thus, condition REVORD can be violated without losing existence of equilibria.

Although there are possibly multiple equilibrium allocations attached to the same equilibrium price system, utility allocations in equilibrium only depend on the price system. Also, the chosen order in $\Pi^{i}(\bar{p})$ does not alter resulting utilities.

\footnotetext{
${ }^{2} e^{r}$ denotes the $r$-th unit vector in $\mathbb{R}^{K}$.

${ }^{3}$ In the first case, none of the objects has to be split.
} 


\section{Lemma 2}

Let $p \in \Delta, i \in\{1,2\}$ and $\pi, \bar{\pi}$ be in $\Pi^{i}(p)$. Then for any initial allocation $\left(\omega^{1}, \omega^{2}\right)$, we obtain

$$
U^{i}\left(\varphi^{i, \pi}\left(p, p \omega^{i}\right)\right)=\sum_{k \in \mathcal{K}}\left(\varphi^{i, \pi}\left(p, p \omega^{i}\right)\right)_{k} u^{i}(k)=\sum_{k \in \mathcal{K}}\left(\varphi^{i, \bar{\pi}}\left(p, p \omega^{i}\right)\right)_{k} u^{i}(k)=U^{i}\left(\varphi^{i, \bar{\pi}}\left(p, p \omega^{i}\right)\right) .
$$

The straightforward proof is omitted.

\section{Market Procedures}

In this section we use a market mechanism to solve the objects division problem. The basic idea is to determine a price system that satisfies the REVORD condition. Both agents are initially endowed with either fractions of objects or, equivalently, income. By maximizing individual utility with given prices an equilibrium allocation prevails. We will see that specific prices will result in equilibrium allocations that implement specific bargaining solutions. Thus, we get a decentralization of the conflict and a perspective bargaining solution through prices.

The basic procedure to solve the division conflict is as follows:

The Market Procedure (basic version):

Step 1: Assign to each object $k$ a price $p_{k}$, so that the price system $p$ satisfies condition REVORD.

Step 2: Compute the total value (at prices $p$ ) of the set of objects, i.e. $M:=\sum_{k \in \mathcal{K}} p_{k} \cdot 1$.

Divide the total amount $\mathrm{M}$ among the agents as income $m^{1}$ and $m^{2}$, respectively.

Step 3: Let each agent "buy" goods according to prices $p$ until no money is left over.

By Theorem 1 we know that the final allocation of objects will be an equilibrium allocation of the corresponding market. Therefore, the outcome of this procedure will be an efficient allocation of the goods in $\mathcal{K}$.

In the following, we will use this procedure to provide implementation results for three bargaining solutions: the Perles-Maschler, Kalai-Smorodinsky and discrete Raiffa solution. For the Perles-Maschler solution, we have to apply the above procedure once, whereas the latter bargaining solutions are obtained by iterating the procedure finitely many often. 


\subsection{The Perles-Maschler Procedure}

The bargaining solution introduced by Perles \& Maschler (1981b) (hereafter PM solution) is known to be the only superadditive bargaining solution for two person bargaining games. Superadditivity roughly says that the bargaining solution of a sum of bargaining problems gives each party at least as much utility as the sum of solutions will provide for him. Perles (1982) showed that the superadditivity axiom is incompatible with the presence of more than two persons..$^{4}$

For the class of object division problems, we can get an implementation result for the PM solution by using the above discussed basic market procedure. For this, we will examine a specific price system $p^{P M} \in \Delta$. Clearly, in order to satisfy condition REVORD, the price of an object has to be between the agents' valuations. We define $p_{k}^{P M}$ to be the (normalized) geometric mean of agents' valuations.

\section{Lemma 3}

The price system $p^{P M} \in \Delta$ defined by $p_{k}^{P M}:=\frac{\sqrt{u^{1}(k) u^{2}(k)}}{\sum_{j \in \mathcal{K}} \sqrt{u^{1}(j) u^{2}(j)}} \quad(k \in \mathcal{K})$ satisfies REVORD.

\section{Proof:}

Take arbitrary $\pi \in \Pi^{1}\left(p^{P M}\right)$. We show $\pi^{*} \in \Pi^{2}\left(p^{P M}\right)$. Since $\pi \in \Pi^{1}\left(p^{P M}\right)$ we have for $j, k \in \mathcal{K}$

$$
\begin{aligned}
\pi^{*}(k)>\pi^{*}(j) \Longleftrightarrow \pi(k)<\pi(j) \Longleftrightarrow \frac{u^{1}(k)}{p_{k}} \geq \frac{u^{1}(j)}{p_{j}} \Longleftrightarrow \frac{u^{1}(k)}{\sqrt{u^{1}(k) u^{2}(k)}} \geq \frac{u^{1}(j)}{\sqrt{u^{1}(j) u^{2}(j)}} \\
\Longleftrightarrow \frac{\sqrt{u^{1}(k) u^{2}(k)}}{u^{2}(k)} \geq \frac{\sqrt{u^{1}(j) u^{2}(j)}}{u^{2}(j)} \Longleftrightarrow \frac{u^{2}(k)}{p_{k}} \leq \frac{u^{2}(j)}{p_{j}},
\end{aligned}
$$

which shows $\pi^{*} \in \Pi^{2}$. The remaining implication runs analogously.

From Lemma 1 and Theorem 1 we know that $p^{P M}$ constitutes an equilibrium price system. Hence, we get a solution for the division problem. What are the properties of this solution? It is obvious that the allocation is efficient and gives both agents a higher utility than the initial allocation.

Yet, conflicts may arise when parties do not want to bargain over the whole set of objects but want to split it into several smaller sets and reach an agreement for each set separately. It is well known that the resulting division is in general not Pareto efficient. Moreover, certain solution

\footnotetext{
${ }^{4} \mathrm{He}$ showed that there is no superadditive solution on the whole class of compactly generated bargaining problems.
} 
concepts or procedures may favor one of the agents. For example, by using the "Adjusted Winner" procedure (cf. Brams \& Taylor (1996)) or equivalently the Kalai-Smorodinsky bargaining solution (cf. Kalai \& Smorodinsky (1975)), some agent prefers to determine the solution of the whole problem, whereas the other prefers to split the problem. However, our procedure with prices $p^{P M}$ will avoid such a conflict. In fact, the solution concept generated by our procedure satisfies the superadditivity property.

Let $\mathcal{E}$ be a market with object set $\mathcal{K}$ and let $\left\{\mathcal{K}^{1}, \ldots, K^{L}\right\}$ be a partition of $\mathcal{K}$ into $L$ nonempty subsets. By $\mathcal{E}^{l}$ we denote the sub-market for objects $\mathcal{K}^{l}$. Utility functions, denoted $U^{i, l}$, are corresponding restrictions of $U^{i}$. Initial endowments are also split canonically, i.e. $\omega_{k}^{1, l}=\omega_{k}^{1}$ for $k \in \mathcal{K}^{l}$.

\section{Theorem 2}

Let $\mathcal{E}$ be a market for objects in $\mathcal{K}$.

(i) Let $\mathcal{E}^{l}(l \in L)$ be sub-markets of $\mathcal{E}$ for objects in $\mathcal{K}^{l}$. Then we obtain for $i=1,2$ and $\pi \in \Pi^{i}\left(p^{P M}\right)$

$$
U^{i}\left(\varphi^{i, \pi}\left(p^{P M}, p^{P M} \omega^{i}\right)\right) \geq \sum_{l=i}^{L} U^{i, l}\left(\varphi^{i, \pi}\left(p^{P M}, p^{P M} \omega^{i, l}\right)\right)
$$

(ii) Let $V^{\mathcal{E}}$ denote the bargaining problem arising from $\mathcal{E}$ and let $\bar{\omega}^{1}=\bar{\omega}^{2}=\left(\frac{1}{2}, \ldots \frac{1}{2}\right) \in \mathbb{R}^{K}$ be equal initial endowment. Then $\left(U^{1}\left(\varphi^{1, \pi}\left(p^{P M}, p^{P M} \bar{\omega}^{1}\right), U^{2}\left(\varphi^{2, \pi}\left(p^{P M}, p^{P M} \bar{\omega}^{2}\right)\right) \in \mathbb{R}^{2}\right.\right.$ coincides with the Perles-Maschler bargaining solution of $V^{\mathcal{E}}$.

\section{Proof:}

By Lemma 2 it suffices to show (4) for some $\pi \Pi^{i}\left(p^{P M}\right)$. Note that the allocations in the sub market form an allocation in $\mathcal{E}$, since $\sum_{i=1,2} \sum_{l=1}^{L} \varphi^{i, l, \pi}\left(p^{P M}, p^{P M} \omega^{i, l}\right)=\sum_{i=1,2} \omega^{i}$. Therefore, we only have to show that this allocation is affordable for both agents. Observe that for a good $k \in \mathcal{K}^{l}$ we obtain

$$
p_{k}^{P M}=\frac{\sqrt{u^{1}(k) \cdot u^{2}(k)}}{\sum_{j \in \mathcal{K}} \sqrt{u^{1}(j) \cdot u^{2}(j)}}=\frac{\sum_{j \in \mathcal{K}^{l}} \sqrt{u^{1}(j) \cdot u^{2}(j)}}{\sum_{j \in \mathcal{K}} \sqrt{u^{1}(j) \cdot u^{2}(j)}} \cdot \frac{\sqrt{u^{1}(k) \cdot u^{2}(k)}}{\sum_{j \in \mathcal{K}^{l}} \sqrt{u^{1}(j) \cdot u^{2}(j)}}=: T^{l} \cdot p_{k}^{P M, l} .
$$

Now, we compute

$p^{P M}\left(\sum_{l=1}^{L} \varphi^{i, l, \pi}\left(p^{P M, l}, p^{P M, l} \omega^{i, l}\right)\right)=\sum_{l=1}^{L} T^{l} p^{P M, l} \varphi^{i, l, \pi}\left(p^{P M, l}, p^{P M, l} \omega^{i, l}\right)=\sum_{l=1}^{L} T^{l} p^{P M, l} \omega^{i, l}=p^{P M} \omega^{i}$. 
Because $p^{P M}$ is an equilibrium price, $\varphi^{i, \pi}\left(p^{P M}, p^{P M} \omega^{i}\right)$ maximizes agent i's utility in his budget set and since utilities are additively separable, this shows (4).

We now show the second part. For the market $\mathcal{E}$ let $\mu(\mathcal{E})$ be the utility allocation in equilibrium with identical endowments and relative to prices $p^{P M}$. It is straightforward to see, that for two markets $\mathcal{E}, \mathcal{E}^{\prime}$ with $V^{\mathcal{E}}=V^{\mathcal{E}^{\prime}}$ we obtain $\mu(\mathcal{E})=\mu\left(\mathcal{E}^{\prime}\right)=: \tilde{\mu}\left(V^{\mathcal{E}}\right)$. Hence, $\mu$ depends only on the associated bargaining problem (take the standard representation). $\mu(\mathcal{E})$ is feasible, individual rational and Pareto optimal in $V^{\mathcal{E}}$. Moreover, it is symmetric, since the price system $p^{P M}$ is invariant under relabelings the agents' names. Finally, any positive linear transformation of utility functions does not alter equilibrium allocations. Linearity of utility functions on $\underline{\bar{X}}^{\mathcal{K}}$ implies that $\tilde{\mu}$ is covariant with (affine) linear transformations of utility. Hence, $\tilde{\mu}$ constitutes a bargaining solutions on $\mathcal{U}$. To show superadditivity of $\tilde{\mu}$ take $V^{\mathcal{E}} \in \mathcal{U}$. Any decomposition of $V^{\mathcal{E}}$ into $V^{1}, V^{2} \in \mathcal{U}$ with $V^{1}+V^{2}=V^{\mathcal{E}}$ can be associated with a subdivision of $\mathcal{E}$ into $\mathcal{E}^{1}$ and $\mathcal{E}^{2}$ such that $V^{i}=V^{\mathcal{E}^{i}}(i=1,2)$ holds (take as $\mathcal{E}^{i}$, e.g., the standard market arising from $\left.V^{1}\right)$. The first part shows superadditivity of $\tilde{\mu}$ on $\mathcal{U}$, i.e. $\tilde{\mu}\left(V^{\mathcal{E}}\right) \geq \tilde{\mu}\left(V^{\mathcal{E}^{1}}\right)+\tilde{\mu}\left(V^{\mathcal{E}^{2}}\right)$. Therefore, $\tilde{\mu}$ has to coincide with the only superadditive solution on $\mathcal{U}$, namely the Perles-Maschler solution. Note that partitioning $\mathcal{K}$ into sub-bundles may involve splitting of goods.

Indeed, inequality (4) shows the following. Consider any finite set of object markets with object sets, say $\left\{K^{1}, \ldots K^{L}\right\}$. Let $\psi\left(\mathcal{K}^{l}\right)$ be the utility allocation, arising from application of the market procedure on the set $\mathcal{K}^{l}$ at prices $p^{P M}$ and equal endowments. Clearly, $\psi\left(\mathcal{K}^{l}\right)$ is a Pareto efficient point in the induced bargaining problem $V^{l}$. Then (4) says that the inequality $\psi\left(\bigcup_{l=1}^{L} K^{l}\right) \geq \sum_{l=1}^{L} \psi\left(\mathcal{K}^{l}\right)$ has to hold. Hence, the bargaining solution $\psi$ has to obey the superadditivity axiom and therefore coincides with the PM solution.

In case there is an agreement on dividing the objects by using prices $p^{P M}$, then the superadditivity property guarantees that neither of the parties would want to split the whole bundle of objects and proceed with the sub-bundles separately by using $p^{P M}$ prices.

\section{Example 1}

Suppose agents' evaluations are as follows:

\begin{tabular}{|r|rr|r|rr|cc|} 
Good & $u_{k}^{1}$ & $u_{k}^{2}$ & $p_{k}$ & $\frac{u_{k}^{1}}{p_{k}}$ & $\frac{u_{k}^{2}}{p_{k}}$ & Ranks 1 & Ranks 2 \\
\hline A & 4 & 1 & $\mathbf{2}$ & 2 & $\frac{1}{2}$ & 1 & 5 \\
B & 9 & 4 & $\mathbf{6}$ & $\frac{3}{2}$ & $\frac{2}{3}$ & 2 & 4 \\
C & 3 & 3 & $\mathbf{3}$ & 1 & 1 & 3,4 & 2,3 \\
D & 5 & 5 & $\mathbf{5}$ & 1 & 1 & 3,4 & 2,3 \\
$\mathbf{E}$ & 2 & 8 & $\mathbf{4}$ & $\frac{1}{2}$ & 2 & 5 & 1 \\
\hline \hline
\end{tabular}

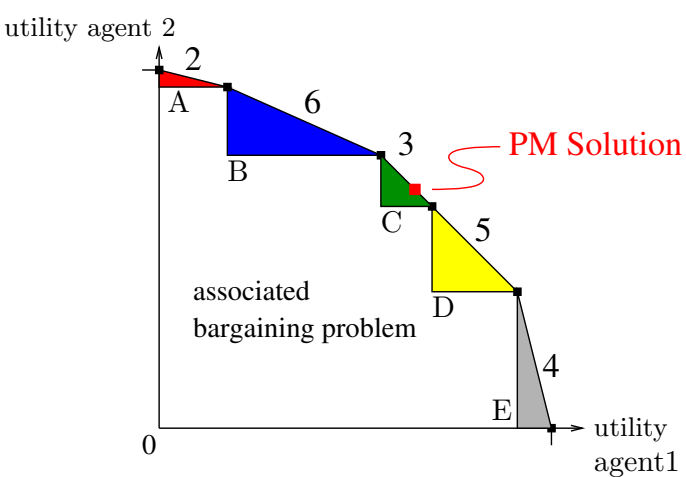


Here $M=20$ and with equal division of $M$ agent 1 receives goods 1,2 and $\frac{2}{3}$ of good 3, whereas agent 2 receives goods 5,4 and $\frac{1}{3}$ of good 3. This is precisely the Perles-Maschler solution of the associated bargaining problem.

\subsection{The Discrete Raiffa Procedure}

Considering the equilibrium price condition REVORD once again, the simplest way to fulfill it is to set prices by either $\hat{p}_{k}^{1}:=u^{1}(k) /\left(\sum_{j} u^{1}(j)\right)$ or $\hat{p}_{k}^{2}:=u^{2}(k) /\left(\sum_{j} u^{2}(j)\right)$. In this case the utility per dollar always equals one for some agent and the equilibrium allocation is basically determined by the other agent's demand. Moreover, we can determine agent $i$ 's utility in equilibrium at prices $\hat{p}^{i}$.

\section{Lemma 4}

In the outcome of the market procedure with prices $\hat{p}^{i}$ and initial incomes $m^{1}, m^{2}$ the utility allocation for agent $i$ is $\frac{m^{1}}{m^{1}+m^{2}} \cdot \sum_{k \in \mathcal{K}} u^{i}(k)$.

\section{Proof:}

Use the fact that agent $i$ 's ratio $\frac{u^{i}(k)}{\hat{p}_{k}^{i}}$ is constant across objects and equals 1 .

The following procedure runs in different rounds. After each round a subset of objects is allocated to the two agents, which remains in their possession during the course of the procedure. However, this could involve splitting an object and allocating a fraction of, say $\lambda$, it to an agent. The remaining fraction will be allocated in subsequent rounds. Therefore, we want to view this remaining fraction of an object as a (complete) object: this is done by multiplying agents' utilities for this object by $1-\lambda$.

As we will now see, the market procedure with likewise prices $\hat{p}^{1}$ and $\hat{p}^{2}$ allows us to determine the discrete Raiffa solution, which was introduced in Raiffa (1953) $)^{5}$. For this, consider following iterative market procedure:

\section{The Discrete Raiffa Procedure:}

Step 0: Define $\mathcal{K}^{0}:=\mathcal{K}$ and $z^{0,1}=z^{0,2}:=0 \in \underline{\bar{X}}^{\mathcal{K}^{0}}$.

We describe how to proceed in round $r+1$ assuming that the set of objects $\mathcal{K}^{r}$ is "on the table".

\footnotetext{
${ }^{5}$ An approximative implementation of the discrete Raiffa Solution can be found in Myerson (1991). Trockel (2004) gives an axiomatization of this bargaining solution
} 
Step $\mathbf{r}+1$ a: Define quantities $M^{r, i}:=\sum_{k \in \mathcal{K}^{r}} u^{i}(k)$ for $i=1,2$. Apply the Market Procedure to $\mathcal{K}^{r}$ with prices $\hat{p}^{1}$ and initial endowments $m^{1}=m^{2}=\frac{1}{2} M^{r, 1}$. This results in an allocation $\left(x^{r, 1}, x^{r, 2}\right)$.

Step $\mathbf{r}+\mathbf{1}$ b: Run the Market Procedure with interchanged roles resulting in an allocation $\left(y^{r, 1}, y^{r, 2}\right)$.

Step $\mathbf{r}+\mathbf{1}$ c: Assign to agent $i$ all (fractions of) objects in $\mathcal{K}^{r}$ that he receives in $x^{i}$ and in $y^{i}$, i.e., assign $z^{r+1,1}:=x^{r+1, i} \wedge y^{r+1, i}(i=1,2)$.

Denote the remaining set of objects by $\mathcal{K}^{r+1}$ and start with step $r+2$, if $\mathcal{K}^{r+1}$ is not empty. Otherwise the procedure is finished.

\section{Theorem 3}

The outcome of the discrete Raiffa procedure is well defined. Moreover, the utility allocation in its outcome coincides with the symmetric discrete Raiffa bargaining solution.

\section{Proof:}

By Lemma 4, the bundle that is assigned to agent $i$ in Step 1 exactly gives him one half of what he thinks the whole set $\mathcal{K}$ is worth. Denote the resulting utilities by $w^{i}=U^{i}\left(z^{1, i}\right)(i=1,2)$. The bargaining problem arising from $\mathcal{K}^{1}$ after Step 1 , is given by the set $\left.V^{1}:=\operatorname{comp} H\left(V^{\mathcal{K}}-w\right) \cap \mathbb{R}_{+}^{2}\right)$. Now the previous step is applied to $\mathcal{K}^{1}$, resp. $V^{1}$. This exactly mimics the construction of the discrete Raiffa solution (in utility space). Therefore, the outcome in utility is well-defined, i.e., the sequence $\left(\left(U^{1}\left(\sum_{s=1}^{r} z^{s, 1}\right), U^{2}\left(\sum_{s=1}^{r} z^{s, 2}\right)\right)\right)_{r=0,1,2, \ldots}$ converges (for $\left.r \rightarrow \infty\right)$ to a Pareto efficient point in $V^{\mathcal{E}}$.

Note that it could happen, that the discrete Raiffa solution is not achievable in a finite number of steps. However, a closer look reveals that this is only the case, when there is a symmetric two objects problem left over, which means $\mathcal{K}^{r}=\{j, k\}$ and $u^{i}(j)=u^{3-i}(k)(i=1,2)$. In that case the procedure could be stopped immediately by assigning and either of the objects should be assigned to that agent that values it most. This means, we could extend the description of the procedure, so that it always runs through at most finitely many rounds.

\subsection{The Kalai-Smorodinsky Procedure}

In the discrete Raiffa procedure, the solution is reached by iteratively assigning bundles to the agents, so that each gets a half share (in utility) of the present object set. In terms of the markets behind, this is achieved by assigning half split of the total value as incomes. Thereby, 
the ratio of agent 2's utility gain to agent 1's utility gain does in general differ from round to round.

In the Kalai-Smorodinsky bargaining solution (see Kalai \& Smorodinsky (1975) as well as Raiffa (1953)) the ratio of assigned utilities equals the ratio of maximal possible utilities. This ratio occurs (in general) only in the first step of the discrete Raiffa procedure. However, with appropriate adjustments of income during the course of the procedure, we can end up with the Kalai-Smorodinsky solution.

\section{The Kalai-Smorodinsky Procedure:}

Step 0: Define $\mathcal{K}^{0}:=\mathcal{K}$ and $z^{0,1}=z^{0,2}:=0 \in \underline{X}^{\mathcal{K}^{0}}$.

Again, we describe how to proceed in round $r+1$ assuming that the set of objects $\mathcal{K}^{r}$ is "on the table".

Step $\mathbf{r}+1$ a: Define quantities $M^{r, i}:=\sum_{k \in \mathcal{K}^{r}} u^{i}(k)$ for $i=1,2$ and denote by $\gamma$ the ration $\gamma:=\frac{M^{0,2}}{M^{0,1}}$. Apply the Market Procedure to $\mathcal{K}^{r}$ with prices $\hat{p}^{1}$ and initial endowments $m^{1}=\frac{M^{r, 2}}{\gamma M^{r, 1}+M^{r, 2}} M^{r, 1}$ and $m^{2}:=M^{r, 1}-m^{1}$. This results in an allocation $\left(x^{r, 1}, x^{r, 2}\right)$.

Step $\mathbf{r}+\mathbf{1}$ b: Run the Market Procedure with interchanged roles resulting in an allocation $\left(y^{r, 1}, y^{r, 2}\right)$. Initial incomes are here $m^{2}:=\frac{\gamma M^{r, 1}}{\gamma M^{r, 1}+M^{r, 2}} M^{r, 2}$ and $m^{1}:=M^{r, 2}-m^{2}$.

Step $\mathbf{r}+1$ c: Assign to agent $i$ all (fractions of) objects in $\mathcal{K}^{r}$ that he receives in $x^{i}$ and in $y^{i}$, i.e., assign $z^{r+1,1}:=x^{r+1, i} \wedge y^{r+1, i}(i=1,2)$.

Denote the remaining set of objects by $\mathcal{K}^{r+1}$ and start with step $r+2$, if $\mathcal{K}^{r+1}$ is not empty. Otherwise the procedure is finished.

\section{Theorem 4}

The Kalai-Smorodinsky procedure is well defined. Moreover, the utility allocation in its outcome coincides with the Kalai-Smorodinsky bargaining solution of the underlying bargaining problem.

\section{Proof:}

Similar to the proof of Theorem 3 the procedure mimics one way to construct the KS solution. Therefore we have to show that the ratio of utility gains in each round is constant and equals the ratio $\frac{M^{0,2}}{M^{0,1}}$. To see this, consider the object division problem in Step $r+1$. According to Lemma 4, agent 1's additional utility is exactly $m^{1}$ as specified in Step $\mathrm{r}+1$ a, whereas player 2's utility gain is $m^{2}$ as specified in Step $\mathrm{r}+1 \mathrm{~b}$. Therefore, the gain ratio amounts to

$$
\frac{m^{2}}{m^{1}}=\frac{\frac{\gamma M^{r, 1}}{\gamma M^{r, 1}+M^{r, 2}} M^{r, 2}}{\frac{M^{r, 2}}{\gamma M^{r, 1}+M^{r, 2}} M^{r, 1}}=\gamma=\frac{M^{0,2}}{M^{0,1}}
$$


This means, the ratio of utility gains in each step is constant and equals the ratio of maximal possible utilities in $V^{\mathcal{E}}$. Therefore, all intermediate utility allocation (and hence the one in the outcome of the procedure) exhibit a ratio of $\frac{M^{0,2}}{M^{0,1}}$, which shows that the final utility allocation coincides with the Kalai-Smorodinsky solution. Note that the sequence of aggregated utility allocations $\left(U^{i}\left(\sum_{s=1}^{r} z^{r, i}\right)\right)_{r=0,1,2 \ldots}(i=1,2)$ is strictly monotonic (in $\left.\mathbb{R}^{2}\right)$ and therefore has to converge to a Pareto efficient point in $V^{\mathcal{E}}$.

Like the discrete Raiffa procedure, this procedure may not converge in finitely many steps. Again, this only happens, when the final solution does not involve splitting of a good. In that case, similar symmetries hold for the last two goods, i.e., $\mathcal{K}^{r}=\{i, j\}$ with $u^{2}(j) / u^{1}(j)<$ $u^{2}(k) / u^{1}(k)$ and $u^{2}(k)=\gamma u^{1}(j)$. Here we can terminate the procedure immediately by assigning good $j$ to agent 1 and good $k$ to agent 2. Hence, the Kalai-Smorodinsky procedure only requires finitely many steps.

\section{Conclusions}

The assumption that agents' utilities over fractions of objects are linear can be further weakened. For an object one can specify a number of possible splits and then treat these splits separately. For example, an agent may live in an apartment only for a couple of months a year. In the rest of the year the other agent may move in. Then agents shall announce their utility of having the object for $k$ months. Equivalently we can view this as having created 12 objects instead of one. The new objects are titled having the apartment for one, two, three, ... months. Application of the market procedure may then result in sharing the apartment by 5 to 7 months. However, in the new structure, this is not considered as splitting the apartment (one has not split one of the new objects).

In the same way we can solve decision processes over finitely many issues, for each of which an option has to be chosen. A discussion on the structure of such problems is found in Raith \& Welzel (1998).

Asymmetries among the agents can be incorporated in the procedure in two ways. First, the assigned income (or initial endowment) can be unequally distributed, so that the market procedure itself will apparently benefit one agent. Second, the chosen price system may reflect an asymmetry among the agents. Obviously, the price systems $\hat{p}^{1}$, which was used to implement the discrete Raiffa and the Kalai-Smorodinsky solution, will not yield a symmetric solution. This means the choice of object prices is not independent of the agents' labels. This is not the case for the price system $p^{P M}$ and hence we obtain a symmetric bargaining solution. Another choice for a "symmetric price" would be $p_{k}:=\min \left(u^{1}(k), u^{2}(k)\right)$, which satisfies REVORD and 
therefore results in a symmetric solution on $\mathcal{U}$.

A simple consideration shows, that all outcomes of the market procedure with equal endowments satisfy the envy-freeness property. Each agent is endowed with what he thinks is worth half of the whole set of objects. In equilibrium, he will be at least as good off as his initial endowment, hence he does not want to change his assignment with the one of his counterpart. In terms of bargaining solutions, this is exactly the midpoint domination property. Each agent receives at least one half of his maximal possible utility.

The case of bargaining problems that are represented as a finite sum of arbitrary ${ }^{6}$ bargaining problems is treated in Ervig \& Haake (2003). Here a notion of bargaining weight that can be traded is established. In the present context this directly translates into shares of a good that can be traded on the market. There it turns out that the Perles-Maschler solution is the only bargaining solution being consistent with decentralized trade of weights.

Last, one can regard (equilibrium) prices as valuation of an object. Because to each object there is an associated bargaining problem that is generated by a "triangle" in $\mathbb{R}^{2}$, we may think of generating a measure on the class of triangular bargaining problems and hence on $\mathcal{U}$ as it is generated by triangular bargaining problems. Clearly, the "price", or "measure" of an object should not depend on how the whole set of objects looks like. The normalization problem (here the sum of prices have to be 1) can be solved by adding an extra object, which is valued 1 by both agents, and the price of which is normalized to 1 . So the unit simplex serves as reference bargaining problem. Having such a measure, say $\psi$, on $\mathcal{U}$, one can define a bargaining solution (w.r.t. $\psi$ ) as the unique point on the boundary, for which the "bargaining problem above this point" has the same measure as the one "below". Desirable properties for bargaining solutions then translate in properties the measure (or price) has to satisfy. The price $p^{P M}$ does satisfy all properties for a defining a bargaining solution in this way, namely the Perles-Maschler solution. In this spirit, we may consider the market approach discussed in this paper as a justification for the measure used to construct the PM solution.

\section{References}

Brams, S. J. \& D. M. Kilgour (2001): "Competitive Fair Division", Journal of Political Economy, 109(2), 418-443.

Brams, S. J. \& A. D. TAYlor (1996): Fair Division - from cake cutting to dispute resolution. Cambridge University Press, Cambridge.

\footnotetext{
${ }^{6}$ i.e. convex, compactly generated utility possibility sets
} 
Ervig, U. \& C.-J. HaAke (2003): "Trading Bargaining Weights", IMW Working Paper 350, University of Bielefeld.

Kalai, E. \& M. Smorodinsky (1975): "Other Solutions to Nash's Bargaining Problem", Econometrica, 43, 513-518.

Myerson, R. B. (1991): Game Theory: Analysis of Conflict. Harvard University Press.

NAsh, J. F. (1950): "The Bargaining Problem", Econometrica, 18, 155-162.

Perles, M. (1982): "Non-Existence of Super-Additive Solutions for 3-Person Games", International Journal of Game Theory, 11(3/4), 151-161.

Perles, M. \& M. Maschler (1981a): "The Present Status of the Superadditive Solution", in Essays in Game Theory and Mathematical Economics, ed. by R. Aumann et al., pp. 103-110. Bibliographisches Institut, Mannheim.

(1981b): "The Super-Additive Solution for the Nash Bargaining Game", International Journal of Game Theory, 10(3/4), 163-193.

Peters, H. J. M. (1992): Axiomatic Bargaining Game Theory, vol. 9 of Theory and decision library (Series C). Kluwer Academic Publishers, Boston / Dordrecht / London.

RaIffa, H. (1953): "Arbitration Schemes for Generalized Two-Person Games", in Contributions to the Theory of Games, ed. by H. Kuhn \& A. W. Tucker, vol. 1 of Annals of Mathematical Studies 24, pp. 361-387. Princeton University Press, Princeton, NJ.

Raith, M. G. \& A. Welzel (1998): "Adjusted Winner: An Algorithm for Implementing Bargaining Solutions in Multi-Issue Negotiations", IMW Working Paper 295, University of Bielefeld.

Rosenmüller, J. (2000): Game Theory: Stochastics, Information, Strategies and Cooperation, vol. 25 of Theory and Decision Library (Series C). Kluwer Academic Publishers, Boston / Dordrecht / London.

Su, F. E. (1999): "Rental Harmony: Sperner's Lemma in Fair Division", American Mathematical Monthly, 106, 930-942.

Thomson, W. (1980): "Two Characterizations of the Raiffa Solution", Economics Letters, 6, 225-231.

Trockel, W. (2004): "An Axiomatization of the Discrete Raiffa Solution", manuscript, IMW / Bielefeld University. 